\title{
Cooperative Guidance Law for Multiple Near Space Interceptors with Impact Time Control
}

\section{GUO Chao*}

School of Automation, Northwestern Polytechnical University, Xi'an, China

\section{LIANG Xiao-Geng**}

Luoyang Optoelectro Technology Development Center, Luoyang, China

\begin{abstract}
We propose a novel cooperative guidance law design method based on the finite time disturbance observer (FTDO) for multiple near space interceptors (NSIs) with impact time control. Initially, we construct a cooperative guidance model with head pursuit, and employ the FTDO to estimate the system disturbance caused by target maneuvering. We subsequently separate the cooperative guidance process into two stages, and develop the normal acceleration command based on the super-twisting algorithm (STA) and disturbance estimated value, to ensure the convergence of the relative distance. Then, we also design the acceleration command along the line-of-sight (LOS), based on the nonsingular fast terminal sliding mode (NFTSM) control, to ensure that all the NSIs simultaneously hit the target. Furthermore, we prove the stability of the closedloop guidance system, based on the Lyapunov theory. Finally, our simulation results of a three-to-one interception scenario show that the proposed cooperative guidance scheme makes all the NSIs hit the target at the same time.
\end{abstract}

Key words: Cooperative guidance law; Finite time disturbance observer (FTDO), Super-twisting algorithm (STA), Nonsingular fast terminal sliding mode (NFTSM)

\section{Introduction}

To deal with the threat of near space air-breathing hypersonic vehicles (NSHV) [1-2], guidance law design for NSI is the most important technology in near space interception systems. The traditional engagement geometry for intercepting high speed targets (NSHV) is typically headon, with very large closing speed. Therefore, the detection precision of a target based on an infrared seeker is usually degraded by aerodynamic heating. To solve this problem, a novel head pursuit engagement geometry, which positions the interceptor ahead of the target on its flight trajectory, has been reported [3-5]. Compared with the head-on geometry, the interceptor can fly in the same direction as the target, and then the closing speed can be significantly reduced, with low energy requirement.
Cooperative attack of multiple missiles has been regarded as an effective interception strategy to deal with the threat of NSHV [6-13]. As a typical cooperative attack scenario, it means that multiple missiles are required to hit the target as simultaneously as possible. Therefore, it is difficult for the NSHV to defend against a group of interceptors at the same time, even though each interceptor has the same guidance performance as the conventional one. An impact-timecontrol guidance (ITCG) law [7] is used to guide multiple missiles to simultaneously hit a stationary target at a desirable impact time. As an extension of study [7], a novel guidance law to control both impact time and impact angle is also proposed in [8], which can be applied for an efficient salvo attack of anti-ship missiles. Based on the work of [9], Zhao proposed a cooperative guidance law of a two-level hierarchical architecture [10], in which both centralized and
This is an Open Access article distributed under the terms of the Creative Commons Attribution Non-Commercial License (http://creativecommons.org/licenses/by$\mathrm{nc} / 3.0 /$ which permits unrestricted non-commercial use, distribution, and reproduction in any medium, provided the original work is properly cited. (c) * Ph. D Student, Corresponding author : dongda20052742@163.com
** Professor 
distributed coordination algorithms are derived, based on the impact-time-control guidance law. A time-constrained cooperative guidance scheme is also developed in [1112], based on the leader-follower method. However, these studies only consider a stationary or low-speed target, and the target maneuvers are neglected. To the best of the authors' knowledge, only a few results have considered a high-speed or maneuvering target intercepted by multiple missiles [13].

To deal with the influence of target maneuver in the guidance system, some advanced guidance methods have been applied, such as the sliding mode guidance (SMG) law [14], and nonlinear Ho guidance law [15]. However, the above methods dealt with the influence of target maneuver at the price of sacrificing normal guidance performance. For example, SMG [14] has strong robustness against system disturbance and parameter uncertainty by designing higher control gain, but chattering phenomenon caused by the discontinuous control law and frequent switching near the sliding surface is inevitable. Therefore, an active disturbance rejection method is imperative to achieve higher guidance performance, for the cooperative interception of multiple NSIs.

Disturbance observer-based control (DOBC) provides an active approach to deal with system disturbances, and improve its robustness (see e.g., [16-19] and the references therein, for a survey of recent development). Initially, it is assumed that there is no system disturbance, or the disturbance is measurable, and a baseline controller is designed; subsequently, a properly disturbance observer is developed to estimate the system disturbance, and the feed-forward compensation term based on the disturbance estimated value is given, to counteract the influence of disturbances. Most of the robust control methods, such as $H_{\infty}$ control [15], are worst case based design, and the disturbance attenuation ability is obtained at the price of degrading normal control performance. However, the DOBC design method can retain normal control performance in the absence of system disturbance, and completely remove the effect of the disturbance from the guidance system, as long as the designed disturbance observer can estimate the disturbance.

Based on the aforementioned analysis, this paper will deal with the cooperative guidance law design problem for multiple NSIs, based on the FTDO. Based on the cooperative guidance model, the cooperative guidance law design is divided into two stages. Firstly, the normal acceleration command is developed, based on the STA and disturbance estimated value. Furthermore, the acceleration command along the LOS is also designed based on the NFTSM, to make all the NSIs simultaneously hit the target. Finally, simulation results show the effectiveness of the proposed cooperative guidance scheme.

Briefly, the rest of this paper is organized as follows. Section 2 introduces the cooperative guidance model for multiple NSIs. Section 3 presents the cooperative guidance law based on the STA and NFTSM. Section 4 gives the simulation results for the multiple NSIs guidance system. Finally, Section 5 draws a brief conclusion.

\section{Problem formulation of cooperative inter- ception}

Figure 1 shows the interceptor-target engagement geometry of head pursuit, where $M$ and $T$ represent the mass center of the interceptor and target, respectively. In contrast with the conventional guidance configuration, the target is located behind the slower NSI. The kinematical equations of the interceptor-target motion can be described as follows:

$$
\left\{\begin{array}{l}
\dot{r}=V_{m} \cos \left(q-\theta_{m}\right)-V_{t} \cos \left(q-\theta_{t}\right) \\
r \dot{q}=-V_{m} \sin \left(q-\theta_{m}\right)+V_{t} \sin \left(q-\theta_{t}\right) \\
a_{m}=V_{m} \dot{\theta}_{m} \\
a_{t}=V_{t} \dot{\theta}_{t}
\end{array}\right.
$$

where, $r$ is the relative distance between the interceptor and target, $q$ denotes the angle between the LOS and the inertial reference line, and $a_{m}$ and $a_{t}$ are the interceptor and target accelerations.

By introducing the variables $V_{r}=\dot{r}$ and $V_{q}=r \dot{q}$, we can obtain:

$$
\left\{\begin{array}{l}
\dot{r}=V_{r} \\
\dot{V}_{r}=-\frac{V_{q}^{2}}{r}+u_{r}-a_{t} \sin \left(q-\theta_{t}\right) \\
\dot{V}_{q}=-\frac{V_{r} V_{q}}{r}+u_{q}-a_{t} \cos \left(q-\theta_{t}\right)
\end{array}\right.
$$

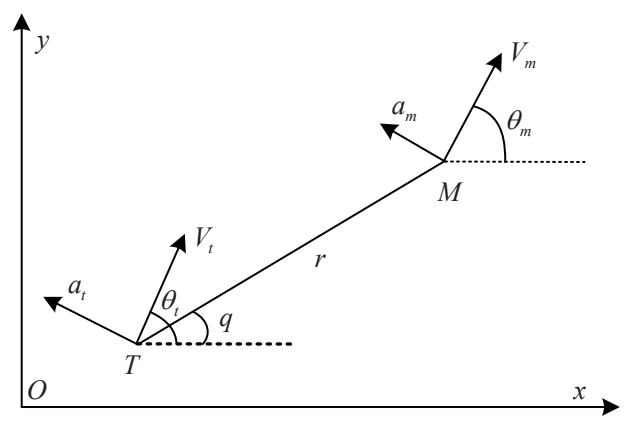

Fig. 1. One-to-one interception geometry 
where, $u_{r}=a_{m} \sin \left(q-\theta_{m}\right)$ and $u_{q}=a_{m} \cos \left(q-\theta_{m}\right)$ denote the accelerations of the NSI in the LOS coordinate system, respectively.

Figure 2 shows the many-to-one engagement geometry with $n$ interceptors. Although the initial condition of each NSI is different from the others, the objective of the cooperative interception is to make all the NSIs hit the target at the same time, as far as possible.

As shown in Fig. 2, the dynamics between the i-th NSI and the target in the LOS coordinate system can be described as follows:

$$
\left\{\begin{array}{l}
\dot{r}_{i}=V_{r i} \\
\dot{V}_{r i}=-\frac{V_{q i}^{2}}{r_{i}}+u_{r i}-a_{t} \sin \left(q_{i}-\theta_{t}\right) \\
\dot{V}_{q i}=-\frac{V_{r i} V_{q i}}{r_{i}}+u_{q i}-a_{t} \cos \left(q_{i}-\theta_{t}\right)
\end{array}\right.
$$

where, $u_{r i}=a_{m i} \sin \left(q_{i}-\theta_{m i}\right)$ and $u_{q i}=a_{m i} \cos \left(q_{i}-\theta_{m i}\right)$.

Based on the above analysis, the cooperative guidance process can be divided into two stages. In order to ensure the convergence of relative distance, i.e., the individual interception can be guaranteed, the normal acceleration command $u_{q i}$ is firstly designed; and then the acceleration command $u_{r i}$ along the LOS is given, to guarantee that all the NSIs simultaneously hit the target.

Remark 1. Since the variation of the closing velocity is relatively small, and the initial closing velocity is smaller than zero, the acceleration command $u_{r i}$ along the LOS is usually set to zero, during the traditional terminal guidance process. Only the normal acceleration command $u_{q i}$ needs to be designed, to send the LOS angular rate to zero. However, in order to control the impact time and implement the cooperative interception, the acceleration command $u_{r i}$ should also be designed.

In this paper, the normal acceleration command

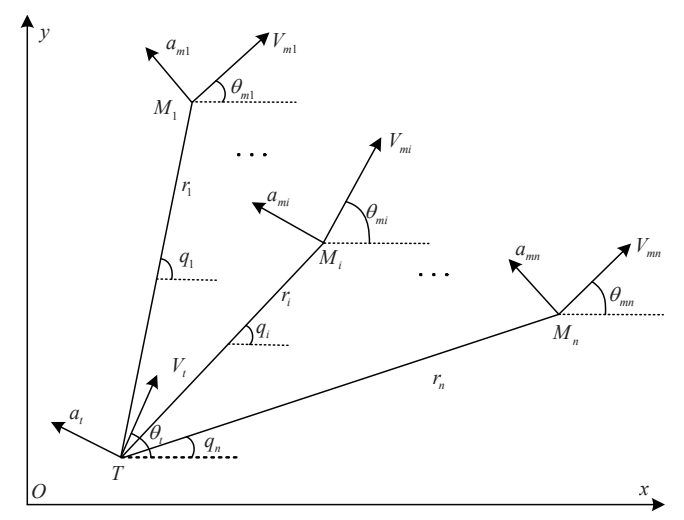

Fig. 2. Many-to-one interception geometry $u_{q i}$ will be given based on the STA and FTDO, and the detailed process can be seen in the next section. Based on reference [20], the impact time control problem can be transformed into the tracking problem of the interceptortarget relative distance. Firstly, a designated interceptortarget relative distance is designed. Furthermore, based on the NFTSM, the acceleration command $u_{r i}$ is given, to make the actual interceptor-target relative distance to track the designated relative distance, i.e., $r_{i} \rightarrow R \rightarrow$ 0 . Then, the objective of the cooperative interception can be successfully obtained. The designated relative distance is given as follows:

$$
R=V_{d}\left(T_{d}-t\right)
$$

where, $T_{d}$ is the designated impact time.

\section{Cooperative guidance law design and sta- bility analysis}

In this subsection, the FTDO is firstly developed to estimate the system disturbance caused by the target maneuver. Then, the acceleration commands $u_{q i}$ and $u_{r i}$ are given, based on the STA and NFTSM, respectively; and the stability analysis is also given.

Define the variables $x_{i 1}=R-r_{i}, x_{i 2}=R-\dot{r}_{i}$, and $x_{i 3}=V_{q i}$; then from (3) and (4) we can obtain:

$$
\left\{\begin{array}{l}
\dot{x}_{i 1}=x_{i 2} \\
\dot{x}_{i 2}=\frac{V_{q i}^{2}}{r_{i}}-u_{r i}-d_{i} \tan \left(q_{i}-\theta_{t}\right) \\
\dot{x}_{i 3}=-\frac{V_{r i} x_{i 3}}{r_{i}}+u_{q i}+d_{i}
\end{array}\right.
$$

where, $d_{i}=-a_{t} \cos \left(q_{i}-\theta_{t}\right)$.

\subsection{FTDO design}

To remove the influence of the system disturbance for the guidance system, the following FTDO is proposed to estimate the disturbance $d_{i}$ :

$$
\left\{\begin{array}{l}
\dot{\bar{z}}_{i 0}=\bar{v}_{i 0}+f_{q i} \\
\bar{v}_{i 0}=-\lambda_{i 0} \underline{L}_{i}^{1 / 3}\left|\bar{z}_{i 0}-x_{i 3}\right|^{2 / 3} \operatorname{sgn}\left(\bar{z}_{i 0}-x_{i 3}\right)-\mu_{0 i}\left(\bar{z}_{i 0}-x_{i 3}\right)+\bar{z}_{i 1} \\
\dot{\bar{z}}_{i 1}=\bar{v}_{i 1} \\
\bar{v}_{i 1}=-\lambda_{i 1} \underline{L}_{i}^{1 / 2}\left|\bar{z}_{i 1}-\bar{v}_{i 0}\right|^{1 / 2} \operatorname{sgn}\left(\bar{z}_{i 1}-\bar{v}_{i 0}\right)-\mu_{1 i}\left(\bar{z}_{i 1}-\bar{v}_{i 0}\right)+\bar{z}_{i 2} \\
\dot{\bar{z}}_{i 2}=\bar{v}_{i 2} \\
\bar{v}_{i 2}=-\lambda_{i 2} \underline{L}_{i}\left|\bar{z}_{i 2}-\bar{v}_{i 1}\right|_{i}^{q_{i} / p_{i}} \operatorname{sgn}\left(\bar{z}_{i 2}-\bar{v}_{i 1}\right)-\mu_{2 i}\left(\bar{z}_{i 2}-\bar{v}_{i 1}\right) \\
\bar{z}_{i 0}=\hat{x}_{i 3}, \bar{z}_{i 1}=\hat{d}_{i}, \bar{z}_{i 2}=\hat{\dot{d}}_{i}
\end{array}\right.
$$


where, $f_{q i}=-\frac{V_{r i} x_{i 3}}{r_{i}}+u_{q i}, \hat{x}_{i 3}$ and $\hat{d}_{i}$ are the estimated values of $x_{i 3}$ and $d_{i i}$, respectively; and $\lambda_{i 0}, \lambda_{i 1}, \lambda_{i 2}, \mu_{i 0}, \mu_{i 1}$ and $\mu_{i 2}$ are the observer gain coefficients to be determined.

Define the variables $\bar{e}_{i 0}=\bar{z}_{i 0}-x_{i 3}, \bar{e}_{i 1}=\bar{z}_{i 1}-d_{i}$, and $\bar{e}_{i 2}=\bar{z}_{i 2}-\dot{d}_{i}$. We can obtain the following disturbance observer error system:

$$
\left\{\begin{array}{l}
\dot{\bar{e}}_{i 0}=-\lambda_{i 0} \underline{L}_{i}^{1 / 3}\left|\bar{e}_{i 0}\right|^{2 / 3} \operatorname{sgn}\left(\bar{e}_{i 0}\right)-\mu_{i 0} \bar{e}_{i 0}+\bar{e}_{i 1} \\
\dot{\bar{e}}_{i 1}=-\lambda_{i 1} \underline{L}_{i}^{1 / 2}\left|\bar{e}_{i 1}-\dot{\bar{e}}_{i 0}\right|^{1 / 2} \operatorname{sgn}\left(\bar{e}_{i 1}-\dot{\bar{e}}_{i 0}\right)-\mu_{i 1}\left(\bar{e}_{i 1}-\dot{\bar{e}}_{i 0}\right)+\bar{e}_{i 2} \\
\dot{\bar{e}}_{i 2}=-\lambda_{i 2} \underline{L}_{i}\left|\bar{e}_{i 2}-\dot{\bar{e}}_{i 1}\right|^{q_{i} / p_{i}} \operatorname{sgn}\left(\bar{e}_{i 2}-\dot{\bar{e}}_{i 1}\right)-\mu_{i 2}\left(\bar{e}_{i 2}-\overline{\bar{e}}_{i 1}\right)-\ddot{d}_{i}
\end{array}\right.
$$

Based on references [21-22], it can be obtained that the error system (7) has finite time stability, i.e., $\bar{z}_{i 1}$ converges to $d_{i}$ in finite time. Furthermore, it is also assumed that the disturbance estimation error satisfies the following constraints:

$$
\left|\bar{e}_{1 i}\right| \leq \delta_{i},\left|\dot{\bar{e}}_{1 i}\right| \leq \bar{\delta}_{i}
$$

where, $\delta_{i}$ and $\bar{\delta}_{i}$ are positive constants.

Remark 2. The parameters $\underline{L}_{i}$ should be chosen large enough to guarantee the finite time convergence of the observation errors. If the Lipshitz constant $\underline{L}_{i}$ is chosen large enough, the dynamic responses of disturbance estimation become faster; and accordingly, large overshoot will also appear. Therefore, the parameter $\underline{L}_{i}$ should be selected in a way that balances the trade off between the response speed and the overshoot.

\subsection{Normal acceleration design}

The following theorem provides a design method for the normal acceleration command $u_{q i}$, based on the STA:

Theorem 1. Consider the nonlinear system (5). The normal acceleration command $u_{q i}$ is designed as follows:

$$
u_{q i}=\frac{V_{r i} x_{i 3}}{r_{i}}-k_{i 3} \varphi_{i 3}\left(x_{i 3}\right)-k_{i 4} \int_{0}^{t} \varphi_{i 4}\left(x_{i 3}\right) \mathrm{d} \tau-\hat{d}_{i}
$$

where,

$$
\begin{aligned}
& \varphi_{i 3}\left(x_{i 3}\right)=\eta_{i 3}\left|x_{i 3}\right|^{1 / 2} \operatorname{sgn}\left(x_{i 3}\right)+\eta_{i 4} x_{i 3}, \quad \eta_{i 3} \geq 1, \eta_{i 4}>0, \\
& \varphi_{i 4}\left(x_{i 3}\right)=\frac{\eta_{i 3}^{2}}{2} \operatorname{sgn}\left(x_{i 3}\right)+\frac{3}{2} \eta_{i 3} \eta_{i 4}\left|x_{i 3}\right|^{1 / 2} \operatorname{sgn}\left(x_{i 3}\right)+\eta_{i 4}^{2} x_{i 3},
\end{aligned}
$$

with,

$$
\left\{\begin{array}{l}
k_{i 3}>\frac{\left[2\left(\chi_{i}+4 \varepsilon_{i}^{2}\right) \varepsilon_{i}+2 \varepsilon_{i}^{2}+2 \bar{\delta}_{i}^{2}+\varepsilon_{i}\right]\left(2 \varepsilon_{i}-1\right)+2 \varepsilon_{i}^{2}}{\chi_{i}\left(2 \varepsilon_{i}-1\right)} \\
k_{i 4}=\chi_{i}+4 \varepsilon_{i}^{2}+2 k_{i 3} \varepsilon_{i} \\
\chi_{i}>0, \varepsilon_{i}>0.5
\end{array},\right.
$$

then, the LOS angular rate can converge to zero.

Proof. From (5) and (9), we can obtain:

$$
\begin{aligned}
\dot{x}_{i 3} & =-k_{i 3} \varphi_{i 3}\left(x_{i 3}\right)-k_{i 4} \int_{0}^{t} \varphi_{i 4}\left(x_{i 3}\right) \mathrm{d} \tau+d_{i}-\hat{d}_{i} \\
& =-k_{i 3} \varphi_{i 3}\left(x_{i 3}\right)-k_{i 4} \int_{0}^{t} \varphi_{i 4}\left(x_{i 3}\right) \mathrm{d} \tau-\bar{e}_{1 i}
\end{aligned}
$$

and,

$$
\begin{aligned}
\varphi_{i 4}\left(x_{i 3}\right) & =\left(\frac{\eta_{i 3}}{2\left|x_{i 3}\right|^{1 / 2}}+\eta_{i 4}\right)\left[\eta_{i 3}\left|x_{i 3}\right|^{1 / 2} \operatorname{sgn}\left(x_{i 3}\right)+\eta_{i 4} x_{i 3}\right] \\
& =\bar{\varphi}_{i 3}\left(x_{i 3}\right) \varphi_{i 3}\left(x_{i 3}\right)
\end{aligned}
$$

where, $\bar{\varphi}_{i 3}\left(x_{i 3}\right)=\frac{\eta_{i 3}}{2\left|x_{i 3}\right|^{1 / 2}}+\eta_{i 4}$.

Define the variables $y_{i 3}=-\bar{e}_{1 i}-k_{i 4} \int_{0}^{t} \varphi_{i 4}\left(x_{i 3}\right) \mathrm{d} \tau$ and $\tilde{\boldsymbol{z}}_{i}=\left[\varphi_{i 3}\left(x_{i 3}\right), y_{i 3}\right]^{T}$. We then have:

$$
\begin{aligned}
& \dot{\tilde{\boldsymbol{z}}}_{i}=\left[\begin{array}{c}
\left(\frac{\eta_{i 3}}{\left|x_{i 3}\right|^{1 / 2}}+\eta_{i 4}\right)\left[-k_{i 3} \varphi_{i 3}\left(x_{i 3}\right)+y_{i 3}\right] \\
-\dot{\bar{e}}_{1 i}-k_{i 4} \varphi_{i 4}\left(x_{i 3}\right)
\end{array}\right] \\
& =\left[\begin{array}{c}
\bar{\varphi}_{i 3}\left(x_{i 3}\right)\left[-k_{i 3} \varphi_{i 3}\left(x_{i 3}\right)+y_{i 3}\right] \\
-\dot{\bar{e}}_{1 i}-k_{i 4} \bar{\varphi}_{i 3}\left(x_{i 3}\right) \varphi_{i 3}\left(x_{i 3}\right)
\end{array}\right] \\
& =\bar{\varphi}_{i 3}\left(x_{i 3}\right)\left[\begin{array}{c}
-k_{i 3} \varphi_{i 3}\left(x_{i 3}\right)+y_{i 3} \\
-\bar{\varphi}_{i 3}^{-1}\left(x_{i 3}\right) \dot{\bar{e}}_{1 i}-k_{i 4} \varphi_{i 3}\left(x_{i 3}\right)
\end{array}\right] \\
& =\bar{\varphi}_{i 3}\left(x_{i 3}\right)\left(\boldsymbol{A}_{i} \tilde{\boldsymbol{z}}_{i}+\boldsymbol{B}_{i} \dot{\hat{e}}_{1 i}\right)
\end{aligned}
$$

where, $\boldsymbol{A}_{i}=\left[\begin{array}{cc}-k_{i 3} & 1 \\ -k_{i 4} & 0\end{array}\right], \boldsymbol{B}_{i}=\left[\begin{array}{c}0 \\ -1\end{array}\right]$, and $\dot{\bar{e}}_{1 i}=\bar{\varphi}_{i 3}^{-1}\left(x_{i 3}\right) \dot{\bar{e}}_{1 i}$.

From (8) and (12), we can also obtain that:

$$
\begin{aligned}
\dot{\hat{e}}_{1 i} \dot{\hat{e}}_{1 i} & =\left(\frac{2\left|x_{i 3}\right|^{1 / 2}}{\eta_{i 3}+2 \eta_{i 4}\left|x_{i 3}\right|^{1 / 2}}\right)^{2} \dot{\bar{e}}_{1 i}^{2} \leq\left(\frac{2\left|x_{i 3}\right|^{1 / 2}}{\eta_{i 3}+2 \eta_{i 4}\left|x_{i 3}\right|^{1 / 2}}\right)^{2} \bar{\delta}_{i}^{2} \\
& \leq 4 \bar{\delta}_{i}^{2}\left|x_{i 3}\right| \leq 4 \bar{\delta}_{i}^{2}\left[\eta_{i 3}\left|x_{i 3}\right|^{1 / 2} \operatorname{sgn}\left(x_{i 3}\right)+\eta_{i 4} x_{i 3}\right]^{2} \\
& =\bar{\delta}_{i}^{2} \tilde{\boldsymbol{z}}_{i}^{T} \boldsymbol{C}_{i}^{T} \boldsymbol{C}_{i} \tilde{\boldsymbol{z}}_{i}
\end{aligned}
$$

where, $\boldsymbol{C}_{i}=\left[\begin{array}{ll}2 & 0\end{array}\right]$.

Consider the following Lyapunov function:

$$
V_{i 1}\left(\tilde{\boldsymbol{z}}_{i}\right)=\tilde{\boldsymbol{z}}_{i}^{T} \boldsymbol{P}_{i} \tilde{\boldsymbol{z}}_{i}
$$

where, $\boldsymbol{P}_{i}=\left[\begin{array}{cc}\chi_{i}+4 \varepsilon_{i}^{2} & -2 \varepsilon_{i} \\ -2 \varepsilon_{i} & 1\end{array}\right]$.

Calculate the derivative of $V_{i 1}\left(\tilde{z}_{i}\right)$ along the trajectory of the system (12). This yields: 


$$
\begin{aligned}
& \dot{V}_{i 1}\left(\tilde{\boldsymbol{z}}_{i}\right)=\dot{\tilde{\boldsymbol{z}}}_{i}^{T} \boldsymbol{P}_{i} \tilde{\boldsymbol{z}}_{i}+\tilde{\boldsymbol{z}}_{i}^{T} \boldsymbol{P}_{i} \dot{\tilde{\boldsymbol{z}}}_{i} \\
& =\left(\boldsymbol{A}_{i} \tilde{z}_{i}+\boldsymbol{B}_{i} \dot{\hat{e}}_{1 i}\right)^{T} \bar{\varphi}_{i}\left(x_{i 3}\right) \boldsymbol{P}_{i} \tilde{z}_{i}+\tilde{z}_{i}^{T} \boldsymbol{P}_{i} \bar{\varphi}_{i}\left(x_{i 3}\right)\left(\boldsymbol{A}_{i} \tilde{z}_{i}+\boldsymbol{B}_{i} \dot{\hat{e}}_{1 i}\right) \\
& =\bar{\varphi}_{i}\left(x_{i 3}\right)\left[\left(\boldsymbol{A}_{i} \tilde{\boldsymbol{z}}_{i}+\boldsymbol{B}_{i} \dot{\hat{e}}_{1 i}\right)^{T} \boldsymbol{P}_{i} \tilde{\boldsymbol{z}}_{i}+\tilde{\boldsymbol{z}}_{i}^{T} \boldsymbol{P}_{i}\left(\boldsymbol{A}_{i} \tilde{\boldsymbol{z}}_{i}+\boldsymbol{B}_{i} \dot{\hat{e}}_{1 i}\right)\right] \\
& =\bar{\varphi}_{i}\left(x_{i 3}\right)\left[\tilde{\boldsymbol{z}}_{i}^{T} \boldsymbol{A}_{i}^{T} \boldsymbol{P}_{i} \tilde{\boldsymbol{z}}_{i}+\dot{\hat{e}}_{1 i}^{T} \boldsymbol{B}_{i}^{T} \boldsymbol{P}_{i} \tilde{\boldsymbol{z}}_{i}+\tilde{\boldsymbol{z}}_{i}^{T} \boldsymbol{P}_{i} \boldsymbol{A}_{i} \tilde{\boldsymbol{z}}_{i}+\tilde{\boldsymbol{z}}_{i}^{T} \boldsymbol{P}_{i} \boldsymbol{B}_{i} \dot{\hat{e}}_{1 i}\right] \\
& \leq \bar{\varphi}_{i}\left(x_{i 3}\right)\left[\tilde{\boldsymbol{z}}_{i}^{T} \boldsymbol{A}_{i}^{T} \boldsymbol{P}_{i} \tilde{z}_{i}+\tilde{\boldsymbol{z}}_{i}^{T} \boldsymbol{P}_{i} \boldsymbol{A}_{i} \tilde{z}_{i}+\tilde{\boldsymbol{z}}_{i}^{T} \boldsymbol{P}_{i} \boldsymbol{B}_{i} \boldsymbol{B}_{i}^{T} \boldsymbol{P}_{i} \tilde{z}_{i}+\dot{\hat{e}}_{1 i} \dot{\hat{e}}_{1 i}\right] \\
& \leq \bar{\varphi}_{i}\left(x_{i 3}\right) \tilde{\boldsymbol{z}}_{i}^{T}\left(\boldsymbol{A}_{i}^{T} \boldsymbol{P}_{i}+\boldsymbol{P}_{i} \boldsymbol{A}_{i}+\boldsymbol{P}_{i} \boldsymbol{B}_{i} \boldsymbol{B}_{i}^{T} \boldsymbol{P}_{i}+\bar{\delta}_{i}^{2} \boldsymbol{C}_{i}^{T} \boldsymbol{C}_{i}\right) \tilde{z}_{i} \\
& =-\bar{\varphi}_{i}\left(x_{i 3}\right) \tilde{\boldsymbol{z}}_{i}^{T} \boldsymbol{Q}_{i} \tilde{z}_{i}
\end{aligned}
$$

where, $\boldsymbol{Q}_{i}=-\left(\boldsymbol{A}_{i}^{T} \boldsymbol{P}_{i}+\boldsymbol{P}_{i} \boldsymbol{A}_{i}+\boldsymbol{P}_{i} \boldsymbol{B}_{i} \boldsymbol{B}_{i}^{T} \boldsymbol{P}_{i}+\bar{\delta}_{i}^{2} \boldsymbol{C}_{i}^{T} \boldsymbol{C}_{i}\right)$.

From (12) and (14), we can obtain:

$$
\boldsymbol{Q}_{i}=\left[\begin{array}{ll}
Q_{i 11} & Q_{i 12} \\
Q_{i 12} & Q_{i 22}
\end{array}\right]
$$

where,

$$
\begin{aligned}
& Q_{i 11}=2 k_{i 3}\left(\chi_{i}+4 \varepsilon_{i}^{2}\right)-4 k_{i 4} \varepsilon_{i}-4 \varepsilon_{i}^{2}-4 \bar{\delta}_{i}^{2}, \\
& Q_{i 12}=-\chi_{i}-4 \varepsilon_{i}^{2}-2 k_{i 3} \varepsilon_{i}+k_{i 4}+2 \varepsilon_{i}, Q_{i 22}=4 \varepsilon_{i}-1 .
\end{aligned}
$$

In order to ensure that $\boldsymbol{Q}_{i}$ is positively defined, select:

$$
k_{i 4}=\chi_{i}+4 \varepsilon_{i}^{2}+2 k_{i 3} \varepsilon_{i}
$$

then, we have:

$$
\begin{aligned}
& \boldsymbol{Q}_{i}-\operatorname{diag}\left\{2 \varepsilon_{i}, 2 \varepsilon_{i}\right\} \\
& =\left[\begin{array}{cc}
2 k_{i 3} \chi_{i}-4\left(\chi_{i}+4 \varepsilon_{i}^{2}\right) \varepsilon_{i}-4 \varepsilon_{i}^{2}-4 \bar{\delta}_{i}^{2}-2 \varepsilon_{i} & 2 \varepsilon_{i} \\
2 \varepsilon_{i} & 2 \varepsilon_{i}-1
\end{array}\right]
\end{aligned}
$$

If the parameters $\chi_{i}$ and $\varepsilon_{i}$ satisfy (9), we can obtain that $\boldsymbol{Q}_{i}$ is positively defined, and satisfies $\lambda_{\text {min }}\left(\boldsymbol{Q}_{i}\right)>2 \varepsilon_{i}$.

From (14) (15) and (18), we have:

$$
\begin{aligned}
\lambda_{\min }\left(\boldsymbol{P}_{i}\right)\left\|\tilde{z}_{i}\right\|_{2}^{2} \leq \tilde{\boldsymbol{z}}_{i}^{T} \boldsymbol{P}_{i} \tilde{z}_{i} \leq \lambda_{\max }\left(\boldsymbol{P}_{i}\right)\left\|\tilde{z}_{i}\right\|_{2}^{2} \\
\dot{V}_{i 1}\left(\tilde{z}_{i}\right) \leq-\bar{\varphi}_{i}\left(x_{i 3}\right) \tilde{z}_{i}^{T} \boldsymbol{Q}_{i} \tilde{z}_{i} \leq-2 \varepsilon_{i} \bar{\varphi}_{i}\left(x_{i 3}\right) \tilde{z}_{i}^{T} \tilde{z}_{i} \\
\leq-2 \varepsilon_{i}\left(\frac{\eta_{i 3}}{2\left|x_{i 3}\right|^{1 / 2}}+\eta_{i 4}\right) \|\left.\tilde{z}_{i}\right|_{2} ^{2} \leq-2 \varepsilon_{i}\left(\frac{\eta_{i 3}}{2\left|x_{i 3}\right|^{1 / 2}}+\eta_{i 4}\right) \frac{V_{i 1}\left(\tilde{z}_{i}\right)}{\lambda_{\max }\left(\boldsymbol{P}_{i}\right)} \\
=-\frac{\varepsilon_{i} \eta_{i 3}}{\left|x_{i 3}\right|^{1 / 2}} \frac{V_{i 1}\left(\tilde{z}_{i}\right)}{\lambda_{\max }\left(\boldsymbol{P}_{i}\right)}-2 \varepsilon_{i} \eta_{i 4} \frac{V_{i 1}\left(\tilde{z}_{i}\right)}{\lambda_{\max }\left(\boldsymbol{P}_{i}\right)} \\
\leq-\varepsilon_{i} \eta_{i 3} \frac{\lambda_{\min }^{1 / 2}\left(\boldsymbol{P}_{i}\right)}{V_{i 1}^{1 / 2}\left(\tilde{z}_{i}\right)} \frac{V_{i 1}\left(\tilde{z}_{i}\right)}{\lambda_{\max }\left(\boldsymbol{P}_{i}\right)}-2 \varepsilon_{i} \eta_{i 4} \frac{V_{i 1}\left(\tilde{z}_{i}\right)}{\lambda_{\max }\left(\boldsymbol{P}_{i}\right)} \\
=-\bar{r}_{i 1} V_{i 1}^{1 / 2}\left(\tilde{z}_{i}\right)-\bar{r}_{i 2} V_{i 1}\left(\tilde{z}_{i}\right)
\end{aligned}
$$

where, $\bar{r}_{i 1}=\varepsilon_{i} \eta_{i 3} \frac{\lambda_{\min }^{1 / 2}\left(\boldsymbol{P}_{i}\right)}{\lambda_{\max }\left(\boldsymbol{P}_{i}\right)}$, and $\bar{r}_{i 2}=\frac{2 \varepsilon_{i} \eta_{i 4}}{\lambda_{\max }\left(\boldsymbol{P}_{i}\right)}$.
By the comparison principle, it is easy to obtain that:

$$
V_{i 1}(t)=\exp \left(-\bar{r}_{i 2} t\right)\left[V_{i 1}^{1 / 2}(0)+\frac{\bar{r}_{i 1}}{\bar{r}_{i 2}}\left(1-\exp \left(\frac{\bar{r}_{i 2} t}{2}\right)\right)\right]^{2}
$$

Therefore, the variables $V_{i 1}(t)$ and $x_{i 3}$ will converge to zero in finite time.

Remark 3. The STA is one of the powerful second order continuous sliding mode control algorithms, which is usually used for systems of relative degree 1. It can generate the continuous control law that drives the sliding mode manifold and its derivative to zero in finite time, in the presence of external disturbance. This algorithm has received much attention, since it was used to design robust exact differentiators and observers [22].

\subsection{Acceleration design along the LOS}

In this section, we propose a design method for the acceleration command $u_{r i}$ along the LOS, which can guarantee that all the NSIs simultaneously hit the target.

Lemma 1. [23] Consider the nonlinear system described by $\dot{x}=f(x) \quad\left(f(0)=0, x \in R^{n}\right)$. Suppose that there exists a smooth positive function $V(x)$ (defined on $U \subset R^{n}$ ), and $\dot{V}(x)+c V^{\alpha}(x)$ is a negative semi-defined function on $U \subset R^{n}$ for $\alpha \in(0,1)$ and $c>0$. Then, the origin is a finite time stable equilibrium, and the settling time satisfies $T\left(x_{0}\right) \leq[c(1-\alpha)]^{-1} V^{1-\alpha}\left(x_{0}\right)$ for all $x_{0}$, in some open neighborhood of the origin.

Define the following sliding mode manifold [24] for the system (5):

$$
s_{i}=x_{i 1}+\bar{\beta}_{i 1}\left|x_{i 1}\right|^{\bar{\gamma}_{i 1}} \operatorname{sgn}\left(x_{i 1}\right)+\bar{\beta}_{i 2}\left|x_{i 2}\right|^{\bar{\gamma}_{i 2}} \operatorname{sgn}\left(x_{i 2}\right)
$$

where, $\quad \bar{\beta}_{i 1}>0, \bar{\beta}_{i 2}>0,1<\bar{\gamma}_{i 2}<2, \quad$ and $\quad \bar{\gamma}_{i 1}>\bar{\gamma}_{i 2}$. Furthermore, a new double power reaching law is designed, as follows [25]:

$$
\dot{s}_{i}=-\left|x_{i 2}\right|^{\bar{\gamma}_{i 2}-1}\left[k_{i 1}\left|s_{i}\right|^{\bar{\alpha}_{i 1}} \operatorname{sgn}\left(s_{i}\right)+k_{i 2}\left|s_{i}\right|^{\bar{\alpha}_{i 2}} \operatorname{sgn}\left(s_{i}\right)\right]
$$

where, $k_{i 1}>0, k_{i 2}>0, \bar{\alpha}_{i 1}>1$, and $0<\bar{\alpha}_{i 2}<1$.

Remark 4. For the sliding mode manifold (22), we can obtain that when the system trajectory is far away from the equilibrium point, the dominant term $\bar{\beta}_{i 1}\left|x_{i 1}\right|^{\bar{\gamma}_{i 1}} \operatorname{sgn}\left(x_{i 1}\right)$ ensures fast convergence. When the system trajectory is close to the origin, the term $x_{i 1}+\bar{\beta}_{i 2}\left|x_{i 2}\right|^{\bar{\gamma}_{i 2}} \operatorname{sgn}\left(x_{i 2}\right)$ plays an important role in the fast convergence of the system state. Compared with the nonsingular terminal sliding mode (NTSM) algorithm, the proposed NFTSM can obtain fast convergence during the whole process. The convergence property of the double power reaching law (23) is similar to the NFTSM, and the analysis process is omitted here. 
Theorem 2. Consider the nonlinear system (5). The acceleration command $u_{r i}$ along the LOS is designed as follows:

$$
\begin{aligned}
u_{r i}= & \frac{\left(1+\bar{\gamma}_{i 1} \bar{\beta}_{i 1}\left|x_{i 1}\right|^{\bar{\gamma}_{i 1}-1}\right)\left|x_{i 2}\right|^{2-\bar{\gamma}_{i 2}} \operatorname{sgn}\left(x_{i 2}\right)}{\bar{\gamma}_{i 2} \bar{\beta}_{i 2}}+\frac{V_{q i}^{2}}{r_{i}}-\hat{d}_{i} \tan \left(q_{i}-\theta_{t}\right) \\
& +\frac{k_{i 1}\left|s_{i}\right|^{\bar{\alpha}_{i 1}} \operatorname{sgn}\left(s_{i}\right)+k_{i 2}\left|s_{i}\right|^{\bar{\alpha}_{i 2}} \operatorname{sgn}\left(s_{i}\right)}{\bar{\gamma}_{i 2} \bar{\beta}_{i 2}}
\end{aligned}
$$

then, all the NSIs hit the target simultaneously. The sliding mode manifold (22) will converge to the neighborhood of $S_{i}=0$, as

$$
\left|s_{i}\right| \leq \Delta_{i}=\min \left\{\left(\frac{\bar{\gamma}_{i 2} \bar{\beta}_{i 2} \tilde{\delta}_{i}}{k_{i 1}}\right)^{1 / \bar{x}_{i 1}},\left(\frac{\bar{\gamma}_{i 2} \bar{\beta}_{i 2} \tilde{\delta}_{i}}{k_{i 2}}\right)^{1 / \bar{x}_{i 2}}\right\}
$$

in finite time. Then, the system state will converge to the following regions:

$$
\left\{\begin{array}{l}
\left|x_{i 1}\right| \leq \min \left\{2 \Delta_{i}, \bar{\beta}_{i 1}^{-1 /\left(\bar{\gamma}_{i 1}+1\right)} \Delta_{i}^{2 /\left(\bar{\gamma}_{i 1}+1\right)}\right\} \\
\left|x_{i 2}\right| \leq\left(\frac{\Delta_{i}}{\bar{\beta}_{i 2}}\right)^{1 / \bar{\gamma}_{i 2}}
\end{array}\right.
$$

Proof. When $x_{i 1} x_{i 2} \neq 0$, the sliding mode manifold (22) can be rewritten as:

$$
S_{i}= \begin{cases}x_{i 1}+\bar{\beta}_{i 1}\left(x_{i 1}\right)^{\bar{\gamma}_{i 1}}+\bar{\beta}_{i 2}\left(x_{i 2}\right)^{\bar{y}_{i 2}} & x_{i 1}>0, x_{i 2}>0 \\ x_{i 1}+\bar{\beta}_{i 1}\left(x_{i 1}\right)^{\bar{\gamma}_{i 1}}-\bar{\beta}_{i 2}\left(-x_{i 2}\right)^{\bar{\gamma}_{i 2}} & x_{i 1}>0, x_{i 2}<0 \\ x_{i 1}-\bar{\beta}_{i 1}\left(-x_{i 1}\right)^{\bar{y}_{i 1}}+\bar{\beta}_{i 2}\left(x_{i 2}\right)^{\bar{y}_{i 2}} & x_{i 1}<0, x_{i 2}>0 \\ x_{i 1}-\bar{\beta}_{i 1}\left(-x_{i 1}\right)^{\bar{y}_{i 1}}-\bar{\beta}_{i 2}\left(-x_{i 2}\right)^{\bar{y}_{i 2}} & x_{i 1}<0, x_{i 2}<0\end{cases}
$$

It can be seen that (27) is obviously continuous and differentiable. Furthermore, we will prove that (22) is still continuous and differentiable, when $x_{i 1} x_{i 2}=0$. The partial derivatives at $x_{i 1}=0$ are:

$$
\begin{aligned}
& \frac{\partial s_{i}\left(x_{i 1}, x_{i 2}\right)}{x_{i 1}}\left(0^{+}, x_{i 2}\right)=1+\lim _{x_{i 1} \rightarrow 0^{+}} \frac{\bar{\beta}_{i 1}\left(x_{i 1}\right)^{\overline{\bar{p}_{11}}}}{x_{i 1}}=1+\lim _{x_{i 1} \rightarrow 0^{+}} \bar{\beta}_{i 1}\left(x_{i 1}\right)^{\bar{\gamma}_{i 1}-1}=1 \\
& \frac{\partial s_{i}\left(x_{i 1}, x_{i 2}\right)}{x_{i 1}}\left(0^{-}, x_{i 2}\right)=1+\lim _{x_{i 1} \rightarrow 0^{-}} \frac{\bar{\beta}_{i 1}\left(-x_{i 1}\right)^{\overline{\gamma_{i 1}}}}{-x_{i 1}}=1+\lim _{x_{i 1} \rightarrow 0^{-}} \bar{\beta}_{i 1}\left(-x_{i 1}\right)^{\bar{y}_{i 1}-1}=1
\end{aligned}
$$

i.e.,

$$
\frac{\partial s_{i}\left(x_{i 1}, x_{i 2}\right)}{x_{i 1}}\left(0^{+}, x_{i 2}\right)=\frac{\partial s_{i}\left(x_{i 1}, x_{i 2}\right)}{x_{i 1}}\left(0^{-}, x_{i 2}\right)
$$

The partial derivatives at $x_{i 2}=0$ are:

$$
\begin{aligned}
& \frac{\partial s_{i}\left(x_{i 1}, x_{i 2}\right)}{x_{i 2}}\left(x_{i 1}, 0^{+}\right)=\lim _{x_{i 2} \rightarrow 0^{+}} \frac{\bar{\beta}_{i 2}\left(x_{i 2}\right)^{\overline{y_{i 2}}}}{x_{i 2}}=\lim _{x_{i 2} \rightarrow 0^{+}} \bar{\beta}_{i 2}\left(x_{i 2}\right)^{\bar{y}_{12}-1}=0 \\
& \frac{\partial s_{i}\left(x_{i 1}, x_{i 2}\right)}{x_{i 2}}\left(x_{i 1}, 0^{-}\right)=\lim _{x_{i 2} \rightarrow 0^{-}} \frac{\bar{\beta}_{i 2}\left(-x_{i 2}\right)^{\overline{y_{i 2}}}}{-x_{i 2}}=\lim _{x_{i 2} \rightarrow 0^{+}} \bar{\beta}_{i 2}\left(-x_{i 2}\right)^{\overline{\bar{y}_{i 2}-1}}=0
\end{aligned}
$$

i.e.,

$$
\frac{\partial s_{i}\left(x_{i 1}, x_{i 2}\right)}{x_{i 2}}\left(x_{i 1}, 0^{+}\right)=\frac{\partial s_{i}\left(x_{i 1}, x_{i 2}\right)}{x_{i 2}}\left(x_{i 1}, 0^{-}\right)
$$

Based on the above analysis, we have from (22):

$\dot{s}_{i}=\dot{x}_{i 1}+\bar{\gamma}_{i 1} \bar{\beta}_{i 1}\left|x_{i 1}\right|^{\bar{\gamma}_{i 1}-1} \dot{x}_{i 1}+\bar{\gamma}_{i 2} \bar{\beta}_{i 2}\left|x_{i 2}\right|^{\bar{\gamma}_{i 2}-1} \dot{x}_{i 2}$

Substitute (24) into (34). This yields:

$$
\begin{aligned}
& \dot{s}_{i}=x_{i 2}+\bar{\gamma}_{i 1} \bar{\beta}_{i 1}\left|x_{i 1}\right|^{\bar{\gamma}_{11}-1} x_{i 2}+\bar{\gamma}_{i 2} \bar{\beta}_{i 2}\left|x_{i 2}\right|^{\mid \bar{Y}_{12}-1}\left[V_{q i}^{2} / r_{i}-u_{r i}-d_{i} \tan \left(q_{i}-\theta_{t}\right)\right] \\
& =\bar{\gamma}_{i 2} \bar{\beta}_{i 2}\left|x_{i 2}\right|^{\bar{\gamma}_{i 2}-1}\left[\hat{d}_{i} \tan \left(q_{i}-\theta_{t}\right)-d_{i} \tan \left(q_{i}-\theta_{t}\right)\right] \\
& -\left|x_{i 2}\right|^{\bar{\gamma}_{12}-1}\left[k_{i 1}\left|s_{i}\right|^{\bar{\alpha}_{i 1}} \operatorname{sgn}\left(s_{i}\right)+k_{i 2}\left|s_{i}\right|^{\bar{\alpha}_{i 2}} \operatorname{sgn}\left(s_{i}\right)\right] \\
& =-\left|x_{i 2}\right|^{\bar{\gamma}_{12}-1}\left[k_{i 1}\left|s_{i}\right|^{\mid \bar{\alpha}_{11}} \operatorname{sgn}\left(s_{i}\right)+k_{i 2}\left|s_{i}\right|^{\bar{\alpha}_{12}} \operatorname{sgn}\left(s_{i}\right)-\bar{\gamma}_{i 2} \bar{\beta}_{i 2} \tilde{e}_{1 i}\right]
\end{aligned}
$$

where, $\tilde{e}_{1 i}=\bar{e}_{1 i} \tan \left(q_{i}-\theta_{t}\right)$. Based on (8), it is also assumed that $\left|\tilde{e}_{1 i}\right| \leq \tilde{\delta}_{i}$, and $\tilde{\delta}_{i}>0$.

Consider the following Lyapunov function:

$$
V_{i 2}=0.5 s_{i}^{2}
$$

Calculate the derivative of $V_{i 2}$ along the trajectory of the system (12). We then have:

$$
\begin{aligned}
\dot{V}_{i 2} & =s_{i} \dot{s}_{i} \\
& =-\left|x_{i 2}\right|^{\bar{\gamma}_{i 2}-1} s_{i}\left[k_{i 1}\left|s_{i}\right|^{\bar{\alpha}_{i 1}} \operatorname{sgn}\left(s_{i}\right)+k_{i 2}\left|s_{i}\right|^{\bar{\alpha}_{i 2}} \operatorname{sgn}\left(s_{i}\right)-\bar{\gamma}_{i 2} \bar{\beta}_{i 2} \tilde{e}_{1 i}\right] \\
& =-\left|x_{i 2}\right|^{\bar{\gamma}_{i 2}-1}\left[k_{i 1}\left|s_{i}\right|^{\bar{\alpha}_{i 1}+1}+k_{i 2}\left|s_{i}\right|^{\bar{\alpha}_{i 2}+1}-\bar{\gamma}_{i 2} \bar{\beta}_{i 2} \tilde{e}_{1 i} s_{i}\right] \\
& \leq-\left|x_{i 2}\right|^{\bar{\gamma}_{i 2}-1}\left[k_{i 1}\left|s_{i}\right|^{\bar{\alpha}_{i 1}+1}+k_{i 2}\left|s_{i}\right|^{\bar{\alpha}_{i 2}+1}-\bar{\gamma}_{i 2} \bar{\beta}_{i 2}\left|s_{i}\right| \cdot \tilde{e}_{1 i} \mid\right]
\end{aligned}
$$

Therefore, (37) can be rewritten as the following two forms:

$$
\begin{aligned}
& \dot{V}_{i 2} \leq-\left|x_{i 2}\right|^{\bar{\gamma}_{i 2}-1}\left[k_{i 1}\left|s_{i}\right|^{\bar{\alpha}_{i 1}+1}+\left|s_{i 2}\right|\left(k_{i 2}\left|s_{i}\right|^{\bar{\alpha}_{i 2}}-\bar{\gamma}_{i 2} \bar{\beta}_{i 2}\left|\tilde{e}_{1 i}\right|\right)\right] \\
& \dot{V}_{i 2} \leq-\left|x_{i 2}\right|^{\bar{\gamma}_{i 2}-1}\left[\left|s_{i}\right|\left(k_{i 1}\left|s_{i}\right|^{\bar{\alpha}_{i 1}}-\bar{\gamma}_{i 2} \bar{\beta}_{i 2}\left|\tilde{e}_{1 i}\right|\right)+k_{i 2}\left|s_{i}\right|^{\bar{\alpha}_{i 2}+1}\right] \\
& \text { For (38), if } k_{i 2}\left|s_{i}\right|^{\bar{\alpha}_{i 2}}-\bar{\gamma}_{i 2} \bar{\beta}_{i 2} \tilde{\delta}_{i} \geq 0 \text {, we have: } \\
& \dot{V}_{i 2} \leq-\left|x_{i 2}\right|^{\bar{\gamma}_{i 2}-1} k_{i 1}\left|s_{i}\right|^{\bar{\alpha}_{i 1}+1} \\
& \quad=-\underline{k}_{i 1}\left|s_{i}\right|^{\bar{\alpha}_{\alpha_{1}+1}}=-2^{\left(\bar{\alpha}_{i 1}+1\right) / 2} \underline{k}_{i 1} V_{i 2}^{\left(\bar{\alpha}_{i 1}+1\right) / 2}
\end{aligned}
$$

where, $\underline{k}_{i 1}=\left|x_{i 2}\right|^{\bar{\gamma}_{i 2}-1} k_{i 1}$.

According to Lemma 1, the settling time can be calculated as:

$$
T_{i 1}=\frac{V_{i}^{\left(1-\bar{\alpha}_{i 1}\right) / 2}(0)}{2^{\left(\bar{\alpha}_{i 1}+1\right) / 2}\left(1-\bar{\alpha}_{i 1}\right) / 2}
$$

i.e., the sliding mode manifold (22) will converge to the following region in finite time $T_{i 1}$ : 
$\left|s_{i}\right| \leq\left(\frac{\bar{\gamma}_{i 2} \bar{\beta}_{i 2} \tilde{\delta}_{i}}{k_{i 2}}\right)^{1 / \bar{\alpha}_{i 2}}$

For (39), if $k_{i 1}\left|s_{i}\right|^{\bar{\alpha}_{i 1}}-\bar{\gamma}_{i 2} \bar{\beta}_{i 2} \tilde{\delta}_{i} \geq 0$, we have

$$
\begin{aligned}
\dot{V}_{i 2} & \leq-\left|x_{i 2}\right|^{\bar{y}_{i 2}-1} k_{i 2}\left|S_{i}\right|^{\bar{\alpha}_{i 2}+1} \\
& =-\underline{k}_{i 2}\left|S_{i}\right|^{\bar{\alpha}_{i 2}+1}=-2^{\left(\bar{\alpha}_{i 2}+1\right) / 2} \underline{k}_{i 2} V_{i 2}^{\left(\bar{\alpha}_{i 2}+1\right) / 2}
\end{aligned}
$$

where, $\underline{k}_{i 2}=\left|x_{i 2}\right|^{\overline{y_{i 2}}-1} k_{i 2}$.

According to Lemma 1, the settling time can also be calculated as:

$$
T_{i 2}=\frac{V_{i}^{\left(1-\bar{\alpha}_{i 2}\right) / 2}(0)}{2^{\left(\bar{\alpha}_{i 2}+1\right) / 2}\left(1-\bar{\alpha}_{i 2}\right) / 2}
$$

i.e., the sliding mode manifold (22) will converge to the following region in finite time $T_{i 2}$ :

$$
\left|s_{i}\right| \leq\left(\frac{\bar{\gamma}_{i 2} \bar{\beta}_{i 2} \tilde{\delta}_{i}}{k_{i 1}}\right)^{1 / \bar{\alpha}_{i 1}}
$$

Based on (42) and (45), we can show that the sliding mode manifold (22) will converge to the region (25) in finite time.

Substitute (24) into (5). This yields:

$$
\begin{aligned}
\dot{x}_{i 2}= & -\frac{\left(1+\bar{\gamma}_{i 1} \bar{\beta}_{i 1}\left|x_{i 1}\right|^{\bar{\gamma}_{i 1}-1}\right)\left|x_{i 2}\right|^{2-\bar{\gamma}_{i 2}} \operatorname{sgn}\left(x_{i 2}\right)}{\bar{\gamma}_{i 2} \bar{\beta}_{i 2}} \\
& -\frac{k_{i 1}\left|s_{i}\right|^{\bar{\alpha}_{i 1}} \operatorname{sgn}\left(s_{i}\right)+k_{i 2}\left|s_{i}\right|^{\bar{\alpha}_{i 2}} \operatorname{sgn}\left(s_{i}\right)-\bar{\gamma}_{i 2} \bar{\beta}_{i 2} \tilde{e}_{1 i}}{\bar{\gamma}_{i 2} \bar{\beta}_{i 2}}
\end{aligned}
$$

Taking $x_{i 2}=0$ into consideration and $\left|s_{i}\right|>\Delta_{i}$, we have:

$$
\dot{x}_{i 2}=\left\{\begin{array}{l}
-\frac{\left[k_{i 1}-\frac{\bar{\gamma}_{i 2} \bar{\beta}_{i 2} \tilde{e}_{1 i}}{\left|s_{i}\right|^{\bar{\alpha}_{i 1}} \operatorname{sgn}\left(s_{i}\right)}\right]\left|s_{i}\right|^{\bar{\alpha}_{i 1}} \operatorname{sgn}\left(s_{i}\right)+k_{i 2}\left|s_{i}\right|^{\bar{\alpha}_{i 2}} \operatorname{sgn}\left(s_{i}\right)}{\bar{\gamma}_{i 2} \bar{\beta}_{i 2}} \neq 0 \\
-\frac{k_{i 1}\left|s_{i}\right|^{\bar{\alpha}_{i 1}} \operatorname{sgn}\left(s_{i}\right)+\left[k_{i 2}-\frac{\bar{\gamma}_{i 2} \bar{\beta}_{i 2} \tilde{e}_{1 i}}{\left|s_{i}\right|^{\bar{\alpha}_{i 2}} \operatorname{sgn}\left(s_{i}\right)}\right]\left|S_{i}\right|^{\bar{\alpha}_{i 2}} \operatorname{sgn}\left(s_{i}\right)}{\bar{\gamma}_{i 2} \bar{\beta}_{i 2}} \neq 0
\end{array}\right.
$$

From (47), we can obtain that the sliding mode manifold (22) will not stay in the region $x_{i 2}=0$, and $\left|s_{i}\right|>\Delta_{i}, x_{i 2}=0$ will not hinder the reachability of the region (25). The sliding mode manifold (22) will converge to the region (25) in finite time. Then, we have:

$$
s_{i}=x_{i 1}+\bar{\beta}_{i 1}\left|x_{i 1}\right|^{\bar{\gamma}_{i 1}} \operatorname{sgn}\left(x_{i 1}\right)+\bar{\beta}_{i 2}\left|x_{i 2}\right|^{\bar{\gamma}_{i 2}} \operatorname{sgn}\left(x_{i 2}\right)=\tau_{i} \leq \Delta_{i}
$$

Furthermore, (48) can also be written in the following form:

$$
x_{i 1}+\bar{\beta}_{i 1}\left|x_{i 1}\right|^{\overline{\gamma_{i}}} \operatorname{sgn}\left(x_{i 1}\right)+\left(\bar{\beta}_{i 2}-\frac{\tau_{i}}{\left|x_{i 2}\right|^{\gamma_{12}} \operatorname{sgn}\left(x_{i 2}\right)}\right)\left|x_{i 2}\right|^{\overline{\gamma_{12}}} \operatorname{sgn}\left(x_{i 2}\right)=0
$$

If $\bar{\beta}_{i 2}-\frac{\tau_{i}}{\left|x_{i 2}\right|^{\bar{Y}_{i 2}} \operatorname{sgn}\left(x_{i 2}\right)}>0$, (49) has a similar form to NFTSM, which also means that the state variable $x_{i 2}$ will converge to the following region:

$$
\left|x_{i 2}\right| \leq\left(\frac{\tau_{i}}{\bar{\beta}_{i 2}}\right)^{1 / \bar{\gamma}_{i 2}} \leq\left(\frac{\Delta_{i}}{\bar{\beta}_{i 2}}\right)^{1 / \bar{\gamma}_{i 2}}
$$

Furthermore, the state variable $x_{i 1}$ will converge to the region:

$\left|x_{i 1}\right| \leq\left|x_{i 1}\right|+\bar{\beta}_{i 1}\left|x_{i 1}\right|^{\bar{\gamma}_{i 1}} \leq \bar{\beta}_{i 2}\left|x_{i 2}\right|^{\bar{\gamma}_{i 2}}+\left|\tau_{i}\right| \leq 2 \Delta_{i}$

It is also known that the following inequality holds:

$\left|x_{i 1}\right|+\bar{\beta}_{i 1}\left|x_{i 1}\right|^{\bar{\gamma}_{i 1}} \geq 2 \sqrt{\bar{\beta}_{i 1}\left|x_{i 1}\right|^{\bar{\gamma}_{i 1}+1}}$

From (51) and (52), we have:

$\left|x_{i 1}\right| \leq \bar{\beta}_{i 1}^{-1 /\left(\bar{\gamma}_{i 1}+1\right)} \Delta_{i}^{2 /\left(\bar{\gamma}_{i 1}+1\right)}$

Therefore, the state variable $x_{i 1}$ will converge to the region:

$\left|x_{i 1}\right| \leq \min \left\{2 \Delta_{i}, \bar{\beta}_{i 1}^{-1 /\left(\bar{\gamma}_{i 1}+1\right)} \Delta_{i}^{2 /\left(\bar{\gamma}_{i 1}+1\right)}\right\}$

The proof is completed.

Remark 5. In practical interception, the normal acceleration command $u_{q i}$ is mainly provided by the aerodynamic force, i.e., the aerodynamic fins. If the aerodynamic force of the NSI is large enough, a high angle of attack can be obtained, and then the acceleration command $u_{r i}$ along the LOS can also be provided by the aerodynamic fins. Otherwise, it is assumed that the angle between the LOS direction and the axial direction of the NSI is small, and then the acceleration command $u_{r i}$ can be provided by the motors on the head and tail of the body [13].

Remark 6. During the implementation, the acceleration of the NSI is bounded according to the following saturation function:

$$
\begin{aligned}
& u_{r i}= \begin{cases}u_{r i \max } \operatorname{sgn}\left(u_{r i}\right) & \text { if }\left|u_{r i}\right| \geq u_{r i \max } \\
u_{r i} & \text { if }\left|u_{r i}\right|<u_{r i \max }\end{cases} \\
& u_{q i}= \begin{cases}u_{q i \max } \operatorname{sgn}\left(u_{q i}\right) & \text { if }\left|u_{q i}\right| \geq u_{q i \max } \\
u_{q i} & \text { if }\left|u_{q i}\right|<u_{q i \max }\end{cases}
\end{aligned}
$$

where, $u_{\text {rimax }}$ and $u_{\text {qimax }}$ are the maximum values of the corresponding acceleration.

\section{Simulation results}

To illustrate the effectiveness of the proposed cooperative 
guidance law, we present in this section some numerical simulations for the multiple NSIs . We consider a threeto-one interception scenario, i.e., three NSIs are used to intercept a moving target. The initial conditions of the NSI and target in inertial coordinate system are shown in Table 1.

The parameters of the FTDO (6) are given as follows:

$\lambda_{i 0}=20, \lambda_{i 1}=1.5, \lambda_{i 2}=1.1, \mu_{i 0}=\mu_{i 2}=0.1, \mu_{i 1}=0.3$,

$q_{i}=0.5, p_{i}=8, \underline{L}_{i}=100, i \in\{1,2,3\}$.

The parameters of the normal acceleration command (9) are selected as follows:

$\eta_{i 3}=1, \eta_{i 4}=0.1, k_{i 3}=3.5, k_{i 4}=21, i \in\{1,2,3\}$.

The parameters of the designated relative distance are given as follows:

$V_{d}=224 \mathrm{~m} / \mathrm{s}, T_{d}=27 \mathrm{~s}$.

In general, the knowledge and experience of experts (including control engineers, operators, etc.) is employed to determine the designated impact time.

Furthermore, the parameters of the acceleration command (24) along the LOS are also chosen as follows:

$$
\begin{aligned}
& \bar{\beta}_{i 1}=1, \quad \bar{\beta}_{i 2}=3, \bar{\gamma}_{i 1}=1.3, \bar{\gamma}_{i 2}=1.2, k_{i 1}=1, \\
& k_{i 2}=2, \quad \bar{\alpha}_{i 1}=1.1, \bar{\alpha}_{i 2}=0.6, i \in\{1,2,3\} .
\end{aligned}
$$

Table 1. Initial conditions of the NSI and target

\begin{tabular}{cccc}
\hline Parameter & Value & Parameter & Value \\
\hline$x_{t 0}$ & $0 \mathrm{~m}$ & $x_{m 0,1}$ & $5908.8 \mathrm{~m}$ \\
$y_{t 0}$ & $0 \mathrm{~m}$ & $y_{m 0,1}$ & $-1041.8 \mathrm{~m}$ \\
$V_{t 0}$ & $1700 \mathrm{~m} / \mathrm{s}$ & $V_{m 0,1}$ & $1500 \mathrm{~m} / \mathrm{s}$ \\
$\theta_{t 0}$ & $0^{\circ}$ & $\theta_{m 0,1}$ & $5^{\circ}$ \\
$x_{m 0,2}$ & $5379.4 \mathrm{~m}$ & $x_{m 0,3}$ & $5416.4 \mathrm{~m}$ \\
$y_{m 0,2}$ & $-470.6 \mathrm{~m}$ & $y_{m 0,3}$ & $955.1 \mathrm{~m}$ \\
$V_{m 0,2}$ & $1500 \mathrm{~m} / \mathrm{s}$ & $V_{m 0,3}$ & $1500 \mathrm{~m} / \mathrm{s}$ \\
$\theta_{m 0,2}$ & $4^{\circ}$ & $\theta_{m 0,3}$ & $-4^{\circ}$ \\
\hline \multicolumn{5}{c}{}
\end{tabular}

The maximum acceleration of the NHSV (X-43 and X-51A) is about $2 \sim 4 \mathrm{~g}$, and the following four interception conditions are selected to test the effectiveness of the proposed cooperative guidance algorithm:

Case 1: No maneuvering target, $a_{t}=0 \mathrm{~m} / \mathrm{s}^{2}$;

Case 2: Step maneuvering target, $a_{t}=10 \mathrm{~m} / \mathrm{s}^{2}$;

Case 3: Sinusoidal maneuvering target, $a_{t}=30 \sin (t) \mathrm{m} / \mathrm{s}^{2}$;

Case 4: The interceptors are launched at the same place with a time interval $0.5 \mathrm{~s}$, and the target acceleration is also $a_{t}=30 \sin (t) \mathrm{m} / \mathrm{s}^{2}$.

The simulation step is $0.001 \mathrm{~s}$. It is assumed that the blind area of the seeker is $150 \mathrm{~m}$, i.e., the guidance system will stop working, when the interceptor-target relative distance satisfies $r_{i}<150 \mathrm{~m}$, and the outputs of the actuators will keep the current values.

Simulation results using the proposed cooperative guidance law based on the FTDO (denoted by CG+FTDO) are firstly presented for the guidance system of multiple NSIs. Then, the SMG is also studied for the simulation comparisons, which does not take into account the impact time control.

The SMG is given as follows:

$$
u_{q i}=\bar{N}_{i} \dot{r}_{i} \dot{q}_{i}-\varepsilon \operatorname{sgn}\left(\dot{q}_{i}\right)
$$

where, $\bar{N}_{i}=6$ and $\varepsilon=200$.

Figure 3 shows the motion trajectory of the three interceptors and target based on the SMG for Case 1. It can be observed that each interceptor has a small miss distance, but the discrepancies in the impact time are large. From Fig. 4(a) and Table 2, we can see that the proposed cooperative guidance law can drive the three interceptors to hit the target simultaneously at the desired impact time. Fig. 4(b) (e) show the curves of the relative distance, tangential velocity, and control inputs of the three interceptors for Case 1. Fig. $5 \sim 10$ and Table 2 show the simulation results for Cases 2 4;

Table 2. Simulation results for multiple NSIs

\begin{tabular}{cccccc}
\hline $\begin{array}{c}\text { Maneuver } \\
\text { type }\end{array}$ & Interceptor & $\begin{array}{c}\text { Interception } \\
\text { Time (s) } \\
\text { /SMG }\end{array}$ & $\begin{array}{c}\text { Miss } \\
\text { Distance (m) } \\
\text { /SMG }\end{array}$ & $\begin{array}{c}\text { Interception } \\
\text { Time (s) } \\
\text { /CG+FTDO }\end{array}$ & $\begin{array}{c}\text { Miss } \\
\text { Distance (m) } \\
\text { /CG+FTDO }\end{array}$ \\
\hline \multirow{3}{*}{ Case 1 } & NSI 1 & 26.747 & 0.011 & 27.000 & 0.011 \\
& NSI 2 & 25.574 & 0.040 & 27.000 & 0.012 \\
& NSI 3 & 25.203 & 0.035 & 27.000 & 0.011 \\
\hline \multirow{3}{*}{ Case 2 } & NSI 1 & 26.742 & 0.086 & 26.993 & 0.052 \\
& NSI 2 & 24.360 & 0.065 & 27.994 & 0.099 \\
& NSI 3 & 21.819 & 0.138 & 26.994 & 0.025 \\
\hline \multirow{3}{*}{ Case 3 } & NSI 1 & 26.774 & 0.779 & 26.996 & 0.055 \\
& NSI 2 & 25.291 & 1.882 & 26.996 & 0.032 \\
& NSI 3 & 24.162 & 0.117 & 26.996 & 0.044 \\
\hline \multirow{3}{*}{ Case 4 } & NSI 1 & 25.603 & 2.029 & 26.997 & 0.204 \\
& NSI 2 & 21.999 & 1.741 & 26.998 & 0.064 \\
& NSI 3 & 18.410 & 1.078 & 26.996 & 0.076 \\
\hline
\end{tabular}


the analysis processes of Cases 2 4 are similar, which are omitted here for brevity.

It is clear from Fig. 3-10 and Table 2 that the proposed guidance law can provide better guidance performance. However, it must be pointed out that we should design the acceleration command $u_{r i}$ along the LOS, to implement the cooperative interception.

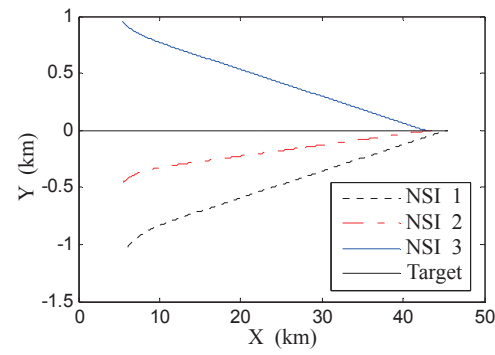

\section{Conclusion}

In this paper, we have dealt with the cooperative interception problem for multiple NSIs with impact time control. The FTDO is introduced to estimate the system disturbance caused by target maneuvering, and the estimated values are employed as feed-forward compensations, to remove the influence of disturbances. Then, the cooperative guidance law design is divided into two stages. Firstly, the normal acceleration command is developed, to guarantee that the tangential velocity of each NSI converges to zero. Secondly, the acceleration command along the LOS is designed, to ensure that all the NSIs simultaneously hit the target. Finally, numerical simulation results on the NSI illustrate the effectiveness and advantage of the proposed cooperative guidance scheme.

Fig. 3. Interceptor-target trajectory by SMG for Case 1

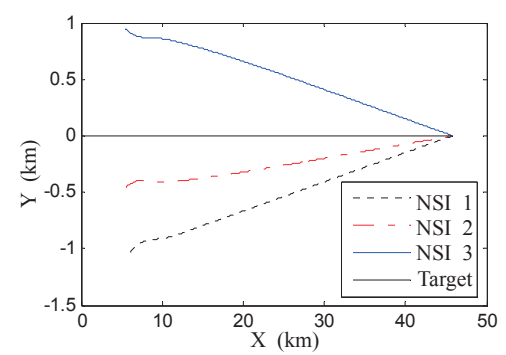

(a) Interceptor-target trajectory by CG+FTDO

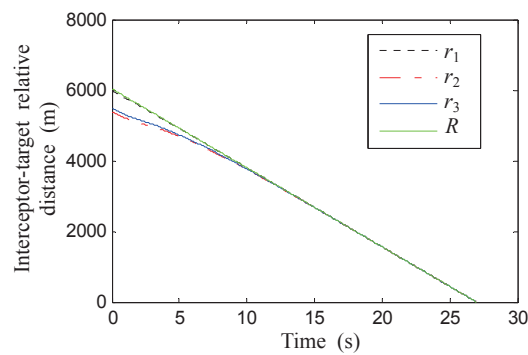

(b) Interceptor-target relative distance

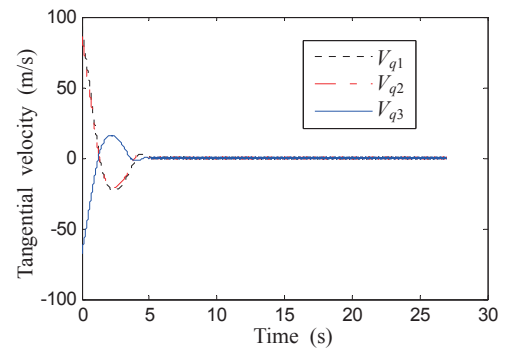

(c) Tangential velocity

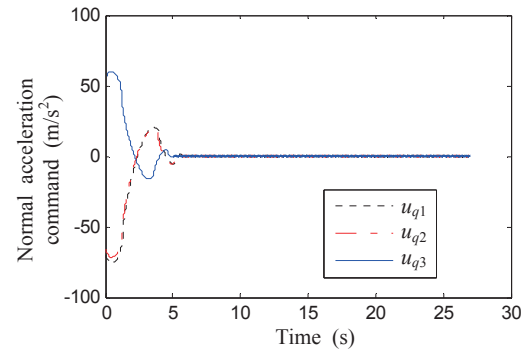

(d) Normal acceleration command

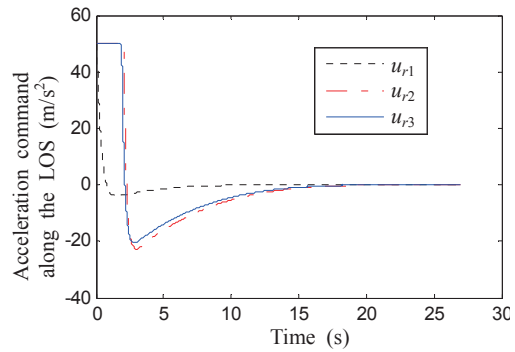

(e) Acceleration command along the LOS

Fig. 4. Simulation results by CG+FTDO for Case 1

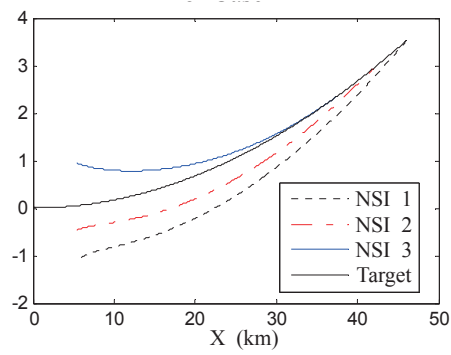

Fig. 5. Interceptor-target trajectory by SMG for Case 2 


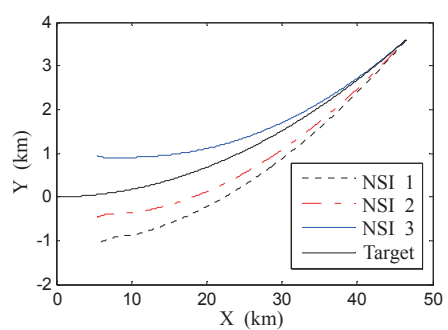

(a) Interceptor-target trajectory by CG+FTDO

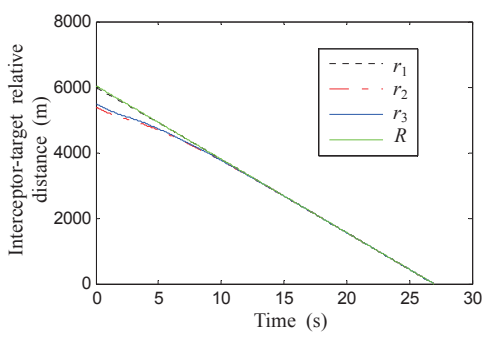

(b) Interceptor-target relative distance

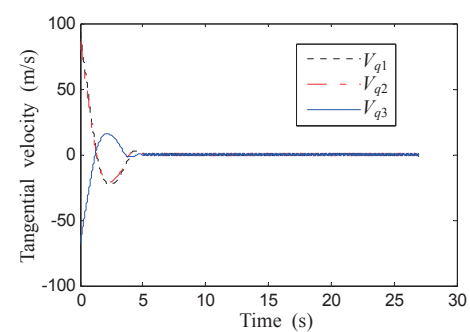

(c) Tangential velocity

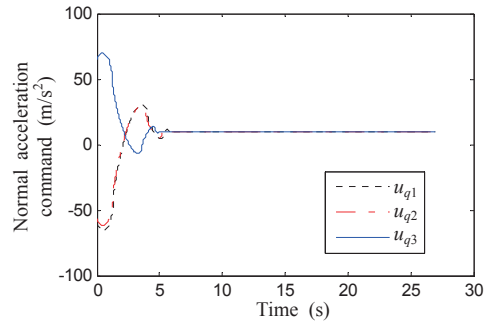

(d) Normal acceleration command

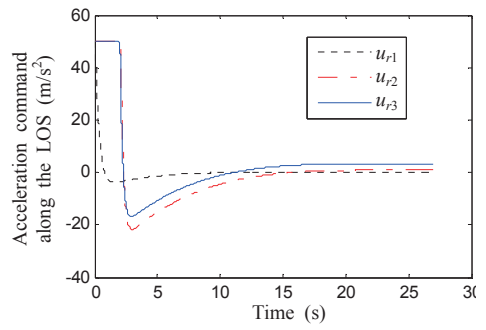

(e) Acceleration command along the LOS

Fig. 6. Simulation results by CG+FTDO for Case 2

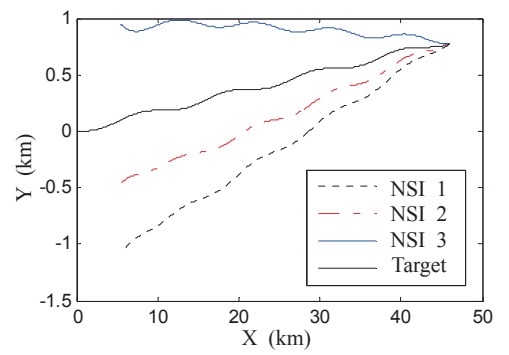

Fig. 7. Interceptor-target trajectory by SMG for Case 3

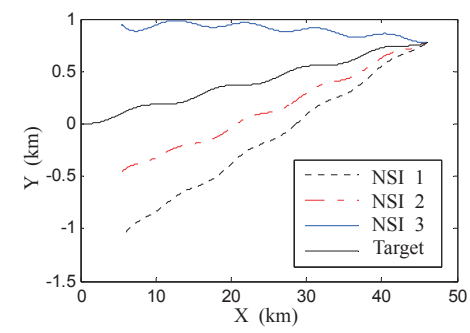

(a) Interceptor-target trajectory by CG+FTDO

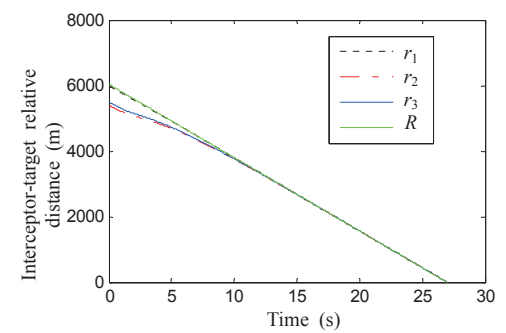

(b) Interceptor-target relative distance

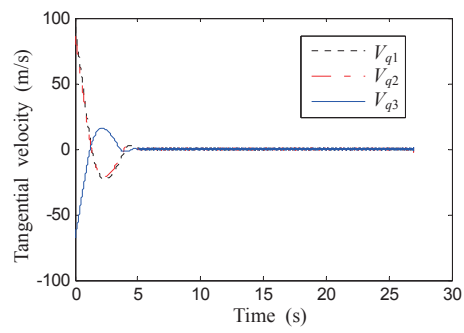

(c) Tangential velocity

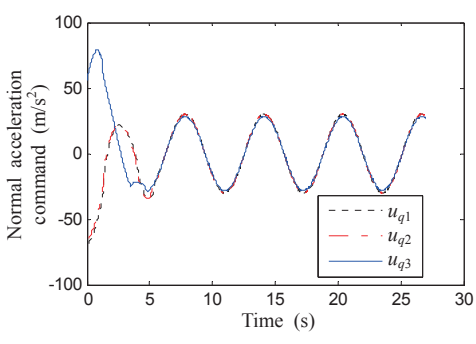

(d) Normal acceleration command

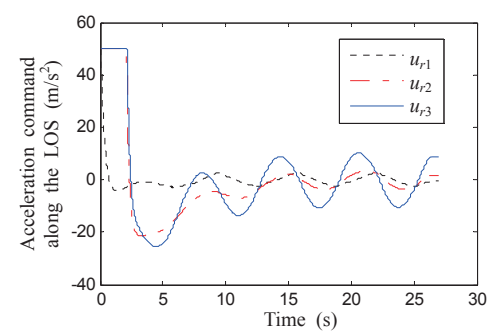

(e) Acceleration command along the LOS

Fig. 8. Simulation results by CG+FTDO for Case 3 


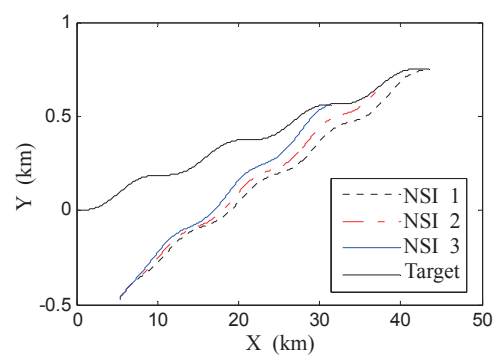

Fig. 9. Interceptor-target trajectory by SMG for Case 4

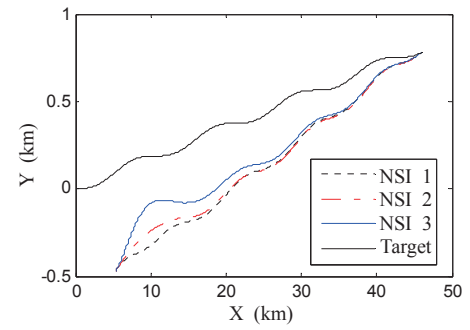

(a) Interceptor-target trajectory by CG+FTDO

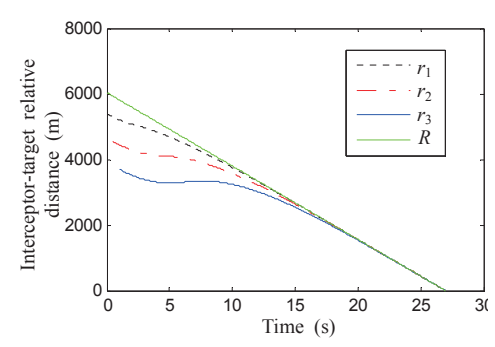

(b) Interceptor-target relative distance

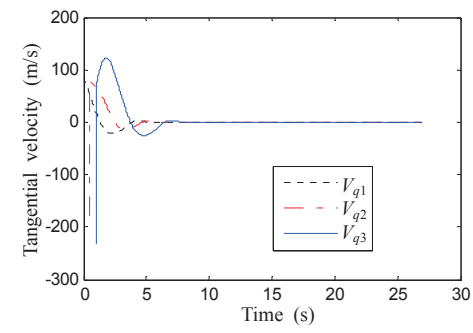

(c) Tangential velocity

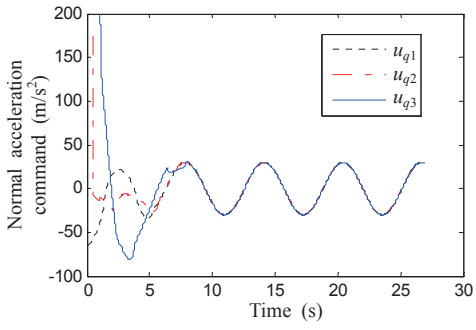

(d) Normal acceleration command

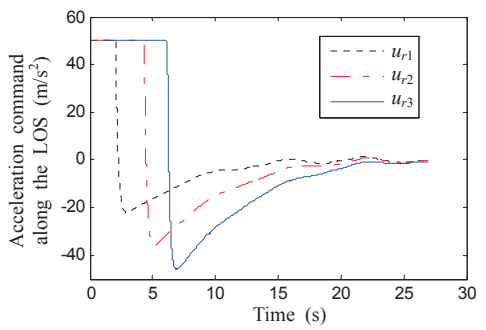

(e) Acceleration command along the LOS

Fig. 10. Simulation results by CG+FTDO for Case 4

\section{References}

[1] Rodriguez, A., Dickeson, J., and Cifdaloz, O., et al., "Modeling and control of scramjet-powered hypersonic vehicles: challenges, trends, \& tradeoffs", In: AIAA Guidance, Navigation and Control Conference and Exhibit, Honolulu, Hawaii, 18-21 August 2008, pp. 2008-6793.

[2] Guo, C., and Liang, X., "Guidance law for near space interceptor based on block backstepping sliding mode and extended state observer", International Journal of Aeronautical and Space Sciences, Vol. 15, No. 2, 2014, pp. 163-172.

[3] Golan, O., and Shima, T., "Head pursuit guidance for hypervelocity interception", In: AIAA Guidance, Navigation and Control Conference and Exhibit, Providence, Rhode Island, 16-19 August 2004, pp. 2004-4885.

[4] Golan, O., and Shima, T., "Precursor interceptor guidance using the sliding mode approach", In: AIAA
Guidance, Navigation and Control Conference and Exhibit, San Francisco, California, 15-18 August 2005, pp. 2005-5965.

[5] Ge, L., Shen, Y., and Gao, Y., et al., "Head pursuit variable structure guidance law for three-dimensional space interception", Chinese Journal of Aeronautics, Vol. 21, No. 3, 2008, pp. 247-251.

[6] Jung, B., and Kim, Y., "Guidance laws for anti-ship missiles using impact angle and impact time", In: AIAA Guidance, Navigation and Control Conference and Exhibit, Keystone, Colorado, 21-24 August 2006, pp. 2006-6432.

[7] Jeon, I., Lee, J., and Tahk, M., "Impact-time-control guidance law for anti-ship missiles", IEEE Transactions on Control Systems Technology, Vol. 14, No. 2, 2006, pp. 260-266.

[8] Lee, J., Jeon, I., and Tahk, M., “Guidance law to control impact time and angle", IEEE Transactions on Aerospace and Electronic Systems, Vol. 43, No. 1, 2007, pp. 301-310.

[9] Jeon, I., Lee, J., and Tahk, M., "Homing guidance law for cooperative attack of multiple missiles", AIAA Journal of 
Guidance, Control and Dynamics, Vol. 33, No. 1, 2010, pp. 275-280.

Zhao, S., and Zhou, R., "Cooperative guidance for multimissile salvo attack", Chinese Journal of Aeronautics, Vol. 21, No. 6, 2008, pp. 533-539.

[10] Zhang, Y., Ma, G., and Wang, X., “Time-cooperative guidance for multi-missiles: a leader-follower strategy. Acta Aeronautica et Astronautica Sinica, Vol. 30, No. 6, 2009, pp. 1109-1118. [in Chinese]

[11] Zhao, S., Zhou, R., and Wei, C., et al., "Design of timeconstrained guidance laws via virtual leader approach", Chinese Journal of Aeronautics, Vol. 23, No. 1, 2010, pp. 103-108.

[12] Zhang, P., Liu, H., and Li, X., et al., "Fault tolerance of cooperative interception using multiple flight vehicles", Journal of the Franklin Institute, Vol. 350, No. 9, 2013, pp. 2373-2395.

[13] Zhou, D., Sun, S., and Teo, K., "Guidance laws with finite time convergence", AIAA Journal of Guidance, Control, and Dynamics, Vol. 32, No. 6, 2009, pp. 1838-1846.

[14] Yang, C., and Chen, H., "Nonlinear Ho guidance law for homing missiles", AIAA Journal of Guidance, Control, and Dynamics, Vol. 21, No. 6, 1998, pp. 882-890.

[15] Chen, W., "Nonlinear disturbance observer-enhanced dynamic inversion control of missiles", AIAA Journal of Guidance, Control, and Dynamics, Vol. 26, No. 1, 2003, pp. 161-166.

[16] Guo, L., and Chen, W., "Disturbance attenuation and rejection for systems with nonlinearity via DOBC approach", International Journal of Robust and Nonlinear Control, Vol.
15, No. 3, 2005, pp. 109-125.

[17] Yang, J., Chen, W., and Li, S., "Non-linear disturbance observer-based robust control for systems with mismatched disturbances/uncertainties", IET Control Theory and Applications, Vol. 5, No. 18, 2011, pp. 2053-2062.

[18] Xia, Y., and Fu, M., Compound control methodology for flight vehicles, Springer Science Business Media, Berlin, 2013.

[19] Zhang, Y., Zhang, Y., and Peng, J., "An impact-timecontrol cooperative guidance law for multi-missiles", Journal of Naval Aeronautical and Astronautical University, Vol. 24, No. 1, 2009, pp. 34-38. [in Chinese]

[20] Levant, A., "High-order sliding models, differentiation and output-feedback control”, International Journal of Control, Vol. 76, No. 9-10, 2003, pp. 924-941.

[21] Shtessel, Y., and Tournes, C., "Integrated higherorder sliding mode guidance and autopilot for dual control missiles", AIAA Journal of Guidance, Control, and Dynamics, Vol. 32, No. 1, 2009, pp. 79-94.

[22] Bhat, S., and Bernstein, D., "Finite-time stability of continuous autonomous systems", SIAM Journal on Control and Optimization, Vol. 38, No. 3, 2000, pp. 751-766.

[23] Yang, L., and Yang, J., "Nonsingular fast terminal sliding-mode control for nonlinear dynamical systems", International Journal of Robust and Nonlinear Control, Vol. 21, No. 16, 2011, pp. 1865-1879.

[24] Zhang, H., Fan, J., and Meng, F., et al., "A new double power reaching law for sliding mode control", Control and Decision, Vol. 28, No. 2, 2013, pp. 289-293. [in Chinese] 